\title{
Reflexe Optomoteur et Activite Tonique Posturale Orthostatique
}

\author{
Par
}

J. B. Baron, J. Cernacek, Z. Niederlandova and N. Ushio

Les relations entre les stimulations rétiniennes et les réponses oculomotrices sont bien connues, décrites depuis longtemps quant à la musculature intrinsèque du globe oculaire, réflexes photomoteurs; par contre elles semblent l'être beaucoup moins quant à la musculature extrinsèque du globe oculaire et leurs relations avec la musculature générale de l'organisme. C'est pourquoi nous voulons remédier à cette lacune en rapportant d'une part notre expérience personnelle (BARON, CERNACEK, NIEDERLANDOVA) et d'autre part celle d'autres auteurs (ZIKMUND) sur ce sujet et en souligner son importance.

Le problème que nous nous étions posés était de savoir comment allait s'organiser l'activité tonique posturale de sujets en bonne santé, présantant une acuité visuelle de $\mathrm{OD}=10 / 10$, $\mathrm{OG}=10 / 10$, sans trouble de l'oculomotricité, placés dans une ambiance obscure, ne reçevant qu'une seule stimulation visuelle représentée par une barre lumineuse verticale placée dans le plan médian du polygone de sustentation à 5 mètres du sujet. La position du sujet nous était donnée par la projection verticale du centre de gravité du corps enregistrée au statokinésimètre ainsi que par une photographie du sujet de dos.

166 sujets masculins et 33 sujets féminins ont été examinés pendant 30 secondes, 3 fois, dans nos conditions expérimentales décrites antérieurement.

\section{RESULTATS}

Le statokinésigramme des sujets examinés nous permet (Fig. 1) de localiser les déplacements antéropostérieurs et latéraux du centre de gravité par rapport au centre du polygone de sustentation et ainsi d'avoir une image de la position du centre de gravité du sujet par rapport au plan médian de son polygone de sustentation où se trouve localisé le stimulus lumineux linéaire. La photographie de dos des sujets permet de déterminer la position du plan médian du sujet par rapport à la barre verticale qui est alignée avec le centre de l'objectif photographique (Fig. 1).

Les résultats sont consignés dans le tableau I. Ils montrent que 19\% des hommes sont localisés en AV/D, 64\% en AR/D, 2\% en AV/G, 15\% en AR/G. 93\% des femmes sont localisées en AR/D. Ce qui est confirmé par la superposition des silhouettes des sujets, soit $83 \%$ à droite pour les hommes et $93 \%$ à droite pour les femmes.

Laboratoire de statokinésimétrie, Centre Psychiatrique Sainte-Anne (Paris, France) 


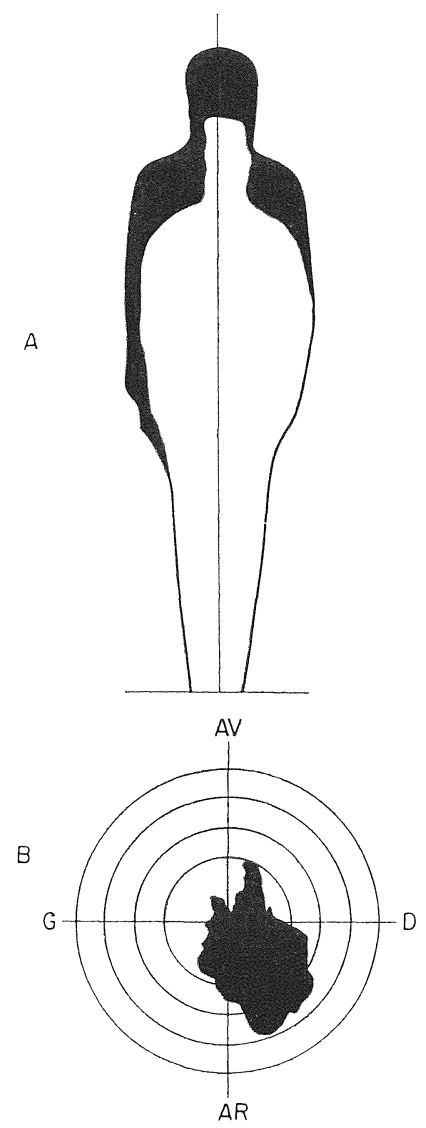

\section{Figure 1}

Superposition de 166:

A: silhouettes provenant de photographies prises de dos

B: statokinésigrammes

de sujets masculins regardant dans une pièce obscure une barre verticale lumineuse située à 5 mètres dans le plan médian du polygone de sustentation.

\section{DISGUSSION}

L'ensemble de ces expériences semble prouver que sur une population de sujets sains (n'ayant aucun trouble de l'acuité visuelle, de l'oculomotricité, ne présentant aucun trouble vestibulaire ni de signe de localisation neurologique), soumis à une sollicitation visuelle d'une barre verticale située dans le plan médian du polygone de sustentation, le schéma corporel allait s'organiser à partir des afférences visuelles provenant d'un seul oeil que nous appelleront oeil directeur de verticalité ce qui allait entrainer une bascule du plan médian du corps du côté opposé.

Il semble que les rétines de l'oeil droit et de l'oeil gauche possèdent des éléments spécifiques sensibles à des stimuli verticaux ou horizontaux à partir desquels les tensions portant sur les différents muscles de la musculature oculomotrice externe vont s'organiser de manière à déterminer un axe horizontal et un axe vertical pour chaque globe oculaire à 1 or $2^{\circ}$ près par rapport aux mêmes coordonnées de l'orbite.

Dans le cas de stimuli verticaux un balayage à dominance verticale dans une limite de 1 à $2^{\circ}$ de déplacement des axes du globe oculaire par rapport à sa position moyenne avex 
Tableau 1

\begin{tabular}{l|c|c|c|c}
\hline & AV-D & AR-D & AV-G & AR-G \\
\hline \multirow{2}{*}{ Hommes } & \multirow{2}{*}{$19 \%$} & $64 \%$ & $2 \%$ & $15 \%$ \\
Femmes & & $93 \%$ & $1.5 \%$ & $5.5 \%$
\end{tabular}

Pourcentage de la localisation des déplacements du centre de gravité (statokinésigramme) par rapport au centre du polygone de sustentation dans les cadrans Avant-Droit (AV-D), Arrière Droit (AR-D), Avant-Gauche, (AV-G), Arrière-Gauche (AR-G) lorsque les sujets (Hommes et Femmes) regardent droit devant eux une barre varticale située dans le plan médian du polygone de sustentation.

\begin{tabular}{l|c|c}
\hline & $\mathrm{D}$ & $\mathrm{G}$ \\
\hline Hommes & $83 \%$ & $17 \%$ \\
Femmes & $93 \%$ & $7 \%$ \\
Femmes & $88 \%$ & $12 \%$ \\
Hommes & &
\end{tabular}

Pourcentage de la localisation (statokinésigramme) à Droite (D) et à Gauche (G) de la même population que précédemment.

dominance d'un oeil par rapport à l'autre, apparait.

Ces différences de tensions correspondant à des déplacements de 1 à $2^{\circ}$ des globes oculaires, entre les muscles moteurs oculaires releveurs et abaisseurs d'un globe oculaire et abducteurs et adducteurs de l'autre vont provoquer des phénomènes de facilitation ou d'inhibition sur l'activité tonique posturale des muscles réglant la posture orthostatique. Facilitation: au niveau des muscles nucaux inclinant le plan sagittal de la tête controlatéralement, au niveau des muscles abducteurs et fléchisseurs du membre inférieur controlatéral fermant l'angle tibio-péronéo-tarsien, et au niveau des adducteurs et extenseurs du membre inférieur homolatéral ouvrant l'angle tibio-péronéo-tarsien, ce qui provoque une inclinaison du plan médian du corps de 1 à $2^{\circ}$ par rapport à la verticale et ses battements autour de cette position, controlatérale a l'oeil dominant de verticalité. En outre il existe un phénomène inhibiteur sur le tonus des muscles symétriques.

Les afférences provenant:

—des éléments spécifiques sensibles à l’horizontalité et la verticalité de la rétine,

—des terminaisons annulo-spiralées des différents nerfs oculomoteurs rendant compte par leur tension de la position des globes oculaires dans l'orbite,

-de la position des labyrinthes dans l'espace réglée d'une part par la tension des muscles nucaux et d'autre part par l'ouverture de l'angle tibio-péronéo-tarsien,

vont permettre de déterminer la hiérarchisation de fonctionnement des éléments oculaires 
oculomoteurs, nucaux, labyrinthiques et des éléments réglant l'amplitude de mouvements de l'articulation tibio-péronéo-tarsienne entre eux et de comprendre les programmes temporospatiaux qui les animent.

Les éléments rapides d'origine proprioceptives oculomotrices visuelles controlent les éléments lents d'origine vestibulaire. L'ensemble du système néanmoins, dépend finalement des qualités visco-élastiques des éléments qui, mécaniquement, règlent les déplacements au niveau de l'articulation tibio-péronéo-tarsienne.

L'introduction d'une erreur de 1 à $2^{\circ}$ entre les différents systèmes permet de comprendre le rattrapage temporal à partir d'informations spatiales différentes dans la programmation des déplacements du centre de gravité.

Il est interéssant de noter que cet oeil de verticalité indépendant des phénomènes de latéralisation classique correspond fonctionnellement à une topographie corticale homolatérale.

\section{BIBLIOGRAPHIE}

-J. B. Baron. - Présentation d'un appareil pour mettre en évidence les déplacements du centre de gravité du corps dans le polygone de sustentation. -Arch. Maladies Profes. 1964. T.25. No 1-2: 41-49.

-J. B. Baron, D. Meziere, Z. Niederlandova, J. G. Raoult. -Participation du système feed-back visuo-oculomoteur dans la régulation de la posture. - Sème Congrés International de Médecine Cybernétique. Naples 1968. 23-29 septembre.

-J. Cernacek, J. B. Baron, J. Jagr. -Eye dominance and body axis. - -IX. Intern. Congress of Neurology and IV. Intern. Congress of Neurosurgery. New. York. 1969. Excerpta Medica Intern. Congr. Ser. $N^{\circ} 193$ (p. 196).

-J. Cernacek, J. Jagr. -Ocular fixation of verticality and vestibular dominance. - International Colloquium on the oculomotor system and brain function. The oculomotor System and Brain Functions. Butterworths. London. 1973. Ed. V. ZIKMUND.: 518-524.

-J. Cernacek, J. Jagr, V. Litvinenkova. -Dominance motrice et station debout. -Rev. Neurol. 1969. 119: $421-422$.

-J. Cernacek, J. Jagr, V. Litvinenkova. -Les facteurs visuo-moteurs influençant la posture. -Activ. Nerv. (Praha) 1971. 13: 32-35.

-Zikmund, J. Balla. - The effect of directional optic stimuli on body gravity centre projection in standing man. -Agressologie. (1973) 14, B: 71-77.

-Z. Niederlandova, V. Litvinenkova. -Dominance d'un hémisphère dans la régulation visuelle de la posture?. -Agressologie. 1976. G (sous presse). 